\title{
Optimal Selection of an Inertial Sensor for Cycle Slip Detection Considering Single-frequency RTK/INS Integrated Navigation*
}

\author{
Younsil Kim, ${ }^{1)}$ Junesol Song, ${ }^{1)}$ Yun Ho, ${ }^{1)}$ Changdon KeE, ${ }^{1 \dagger}$ and Byungwoon PARK ${ }^{2)}$ \\ ${ }^{1)}$ School of Mechanical and Aerospace Engineering and the Institute of Advanced Aerospace Technology, Seoul National University, \\ Seoul 08826, South Korea \\ ${ }^{2)}$ Department of Aerospace Engineering, Sejong University, Seoul 05006, South Korea
}

\begin{abstract}
This paper suggests a methodology for the selection of an inertial sensor for cycle slip detection. The satellitedifference and time-difference residual between the predicted and measured carrier phases is defined as the monitoring value for cycle slip detection. The inertial navigation system (INS) position is used to calculate the predicted observation, and its error mainly contributes to the residual. For one cycle slip detection, the monitoring value residual should be smaller than the threshold. Based on this approach, we derive the maximum permissible position estimation error of the INS to detect one cycle slip. An Earth-centered, Earth-fixed (ECEF) frame-based INS is used for the derivations. Then, we formulate the position estimation error as a function of the inertial sensor specifications using suitable assumptions. Using resulting equation, the optimal inertial sensor can be selected based on the required cycle slip detection performance. To verify the process of optimal IMU selection, simulation data is used. The derived position estimation error of the INS is analyzed carefully, and the monitoring value elicited by the INS error is also investigated. As a result, the selected IMU satisfies the designed cycle slip detection performance within the microelectromechanical systems (MEMS) grade.
\end{abstract}

Key Words: GPS Cycle Slip, MEMS Inertial Sensor, INS

\section{Nomenclature}

$\phi:$ GPS L1 carrier phase observation [m]

$N$ : integer ambiguity

$\lambda$ : GPS L1 carrier phase wavelength [m]

$r$ : magnitude of vector $\boldsymbol{r}[\mathrm{m}]$

$r$ : position vector in ECEF frame $[\mathrm{m}]$

$v$ : velocity vector in ECEF frame $[\mathrm{m} / \mathrm{sec}]$

$\psi$ : attitude vector between body frame and ECEF frame $[\mathrm{rad}]$

$\boldsymbol{e}$ : line-of-sight vector in ECEF frame [m]

$y$ : inertial sensor output vector

$\boldsymbol{b}$ : bias error vector of three-axis inertial sensor

$w$ : Gaussian random noise vector

$f$ : specific-force vector of vehicle in body frame $\left[\mathrm{m} / \mathrm{sec}^{2}\right]$

$\omega$ : angular rate of vehicle in body frame $[\mathrm{rad} / \mathrm{sec}]$

$C_{B}^{E}$ : direction cosine matrix from body frame to ECEF frame

$a$ : magnitude of body acceleration of vehicle $\left[\mathrm{m} / \mathrm{sec}^{2}\right]$

$g_{0}$ : gravitational acceleration $\left[\mathrm{m} / \mathrm{sec}^{2}\right]$

$n$ : number of inertial sensor output samples between consecutive GPS epochs

$T$ : inertial sensor sampling period [sec]

$\delta(\cdot)$ : error value

$\nabla(\cdot)$ : single difference between satellites

$\Delta_{t}(\cdot)$ : difference between consecutive GPS epochs

(C) 2016 The Japan Society for Aeronautical and Space Sciences

*Received 13 May 2015; final revision received 15 January 2016; accepted for publication 23 March 2016.

†Corresponding author, kee@snu.ac.kr
$N\left(m, \sigma^{2}\right)$ : Gaussian distribution with mean $(m)$ and standard deviation $\left(\sigma^{2}\right)$

$[\boldsymbol{V}]^{\times}$: skew symmetric matrix form of arbitrary vector $V$

$\boldsymbol{V}_{1} \cdot \boldsymbol{V}_{2}$ : dot product of vector $\boldsymbol{V}_{1}$ and $\boldsymbol{V}_{2}$

Subscripts

$u$ : user

$a$ : accelerometer

$g$ : gyro

$k$ : epoch index

GPS: produced from GPS

INS: produced from INS

Superscripts

$i, j:$ satellite index

+ : value after GPS measurement update

-: value after INS time update

Embellishment

$$
\begin{aligned}
\hat{\text { : }} & \text { measured value } \\
\text { : } & \text { estimated value } \\
\text { none: } & \text { true value }
\end{aligned}
$$

\section{Introduction}

Currently, m-level positioning accuracy is easily achieved using pseudorange measurements of global positioning system (GPS) satellites and is widely used in many types of industry. However, for cm-level positioning accuracy, the pseudorange measurements are not sufficient because of their large observation errors. For this reason, carrier phase-based positioning is necessary to achieve cm-level accuracy. Because of its complexity and high cost of implementation, cm-level positioning has not yet been popularized as much as m-level 
positioning. Currently, carrier phase-based positioning is widely studied for services demanding high accuracy positioning, such as aircraft navigation and land vehicles.

However, to use the carrier phase as a measurement for positioning, carrier-phase fault detection is performed before the positioning. Cycle slip detection is one of the most important issues in the implementation of a GPS carrier-phase positioning system in a vehicle because cycle slip is very frequent in a weak carrier signal. It results in the degradation of the vehicle's position accuracy due to an incorrect ambiguity resolution.

Cycle slip detection algorithms have been studied and developed for several decades. Many researchers have studied cycle slip detection since the early 1990s. In the capacity of a single-frequency GPS receiver, phase-code comparison, phase-phase ionospheric residual, doppler integration, and differential phases of time methods exist. ${ }^{1)}$ In addition, Kalman filtering has also been used to detect cycle slips. ${ }^{2}$ However, these methods can only handle a few cycle slips safely. In the capacity of a dual-frequency GPS receiver, Bisnath and Langley, ${ }^{3)}$ Blewitt, ${ }^{4)}$ and Gao et al. ${ }^{5,6)}$ have proposed cycle slip detection based on L1 and L2 carrier-phase measurements. Recently, Banville and Langley, ${ }^{7)}$ Cai et al., ${ }^{8)}$ and $\mathrm{Liu}^{9)}$ suggested algorithms that are robust in high ionospheric activity. However, only a multiple-frequency receiver can utilize these algorithms. Consequently, the overall system cost could be expensive.

To overcome these limitations, an inertial navigation system (INS) is occasionally integrated with a GPS to detect cycle slips. The INS-predicted carrier phase is equivalent to the measured carrier phase, so that the cycle slip can be detected by comparing the two values. With the INS, the carrier phase can be predicted more precisely, especially in a highly dynamic situation. This enables the cycle slip detection performance to be improved. It is also advantageous that this method can be implemented in the capacity of a singlefrequency receiver. Altmayer, ${ }^{10)}$ Colombo et al., ${ }^{11)}$ Takasu and Yasuda, ${ }^{12)}$ and Du and $\mathrm{Gao}^{13,14)}$ used GPS/INS integrated systems to detect cycle slips. Furthermore, Song et al. ${ }^{15)}$ investigated the allowable odometer and gyro sensor error limit for cycle slip detection. In this algorithm, the INS position estimation accuracy mainly contributes to the cycle slip detection performance. Thus, for an optimal system design based on performance and cost, proper inertial sensor selection is critical. The inertial sensor cost and performance vary greatly according to the grade.

Therefore, in this paper, we discuss the optimal selection of an inertial sensor for cycle slip detection, especially in the case of single-frequency real-time kinematic (RTK)/ INS integrated navigation. The satellite-difference (SD) and time-difference (TD) residual between the predicted and measured carrier phases is defined as the monitoring value for cycle slip detection. RTK/INS integrated navigation is used to calculate the predicted observation. To select a proper inertial sensor, a theoretical analysis is conducted. We formulate the cycle slip detection performance for inertial sensor specifications. The INS output errors were investigated to determine the upper bound of the estimated position error by considering the cycle slip detection performance. As a result, we can predict the cycle slip performance according to gyro and accelerometer selection. By selecting an IMU that elicits the upper bound of INS accuracy for cycle slip detection, we can satisfy both required cycle slip performance and cost-effectiveness. To verify the selection process, simulation data is used. In addition, to verify the assumptions that are made during the formulation, static experiments were conducted. As a result, we could verify the suggested selection process.

\section{Cycle Slip Detection Algorithm and Error Analysis}

\subsection{Cycle slip detection algorithm}

To detect cycle slip, the satellite-difference and timedifference residual between the measured and predicted carrier phases is defined as the monitoring value for cycle slip detection. Unlike conventional cycle slip detection algorithms that use linear combinations of multiple-frequency GPS observations, this cycle slip detection algorithm uses only singlefrequency GPS observations. Therefore, the proposed algorithm can detect cycle slip in any case, regardless of the size of simultaneous cycle slip in multiple frequencies.

To derive the monitoring value, we use the time and satellite difference of the carrier-phase measurement. In this process, clock bias terms, atmosphere-related errors, and orbit errors are greatly reduced. ${ }^{15,16)}$

Then, the resulting monitoring value can be defined as follows.

$$
\bar{M}_{k+1}^{i}={ }^{i} \nabla^{j} \Delta_{t} \hat{\phi}_{\mathrm{GPS}}-{ }^{i} \nabla^{j} \Delta_{t} \bar{\phi}_{\mathrm{INS}}
$$

The reason why differenced carrier-phase measurements are considered instead of un-differenced carrier-phase measurements is that RTK algorithms are usually based on double-differenced (satellite and receiver-difference) carrierphase measurements to compensate for most of the measurement errors. ${ }^{10)}$ Hence, in this paper, the cycle slip detected by the monitoring value implies the satellite-differenced value instead of the un-differenced one.

The prediction of the carrier-phase term in Eq. (1) is performed using INS. The INS estimates the position, velocity, and attitude of the vehicle. To detect the cycle slip at the $k+1$ epoch, the inertial sensor measurements between $k$ and $k+1$ epoch, velocity and attitude at $k$ epoch are used.

Then, by calculating the monitoring value at each epoch, cycle slip can be detected as follows.

$$
\bar{M}_{k+1}^{i}\left\{\begin{array}{lll}
<\text { threshold } & \rightarrow & \text { no cycle slip } \\
\geq \text { threshold } & \rightarrow & \text { cycle slip }
\end{array}\right.
$$

The cycle slip performance is generally expressed through the minimum detectable cycle slip. In addition, the monitoring value error determines the resolution of cycle slip detection.

The threshold of the monitoring value is different from the required cycle slip detection performance. The threshold for the cycle slip detection can be determined from the aspect of false alarm and miss detection probability. ${ }^{15)}$ 


\subsection{Monitoring value error analysis}

To analyze the maximum permissible range estimation error in order to detect one cycle slip, the error analysis of the monitoring value is performed as follows.

$$
\begin{aligned}
& \bar{M}_{k+1}^{i} \\
& \quad={ }^{i} \nabla^{j} \Delta_{t} \hat{\phi}_{\mathrm{GPS}}-{ }^{i} \nabla^{j} \Delta_{t} \bar{\phi}_{\mathrm{INS}} \\
& =\left({ }^{i} \nabla^{j} \Delta_{t} \hat{\phi}_{\mathrm{GPS}}-{ }^{i} \nabla^{j} \Delta_{t} \phi\right)-\left({ }^{i} \nabla^{j} \Delta_{t} \bar{\phi}_{\mathrm{INS}}-{ }^{i} \nabla^{j} \Delta_{t} \phi\right) \\
& =\delta^{i} \nabla^{j} \Delta_{t} \phi_{\mathrm{GPS}}-\delta^{i} \nabla^{j} \Delta_{t} \phi_{\mathrm{INS}}
\end{aligned}
$$

The $\phi$ term is the true value of the carrier phase and is the same as the range between the satellite and user.

Before further derivation, the definitions of the symbols are represented graphically. Figuer 1 presents the user and satellite configuration during consecutive GPS epochs. Each of the terms represented in Fig. 1 is used to express the error equation of the monitoring value.

Using the graphical representation of the user and satellite geometry, the true value of the differenced carrier phase in Eq. (2) can be expressed as follows.

$$
\begin{aligned}
{ }^{i} \nabla^{j} \Delta_{t} \phi= & \left(\boldsymbol{r}_{k+1}^{i}-\boldsymbol{r}_{u, k+1}\right) \cdot \boldsymbol{e}_{k+1}^{i}-\left(\boldsymbol{r}_{k}^{i}-\boldsymbol{r}_{u, k}\right) \cdot \boldsymbol{e}_{k}^{i} \\
& -\left(\boldsymbol{r}_{k+1}^{j}-\boldsymbol{r}_{u, k+1}\right) \cdot \boldsymbol{e}_{k+1}^{j}+\left(\boldsymbol{r}_{k}^{j}-\boldsymbol{r}_{u, k}\right) \cdot \boldsymbol{e}_{k}^{j}
\end{aligned}
$$

Then, we analyze the errors in Eq. (2) term-by-term. First, the measurement error of the GPS carrier phase is investigated.

$$
\begin{aligned}
\delta^{i} \nabla^{j} \Delta_{t} \phi_{\mathrm{GPS}} & ={ }^{i} \nabla^{j} \Delta_{t} \hat{\phi}_{\mathrm{GPS}}-{ }^{i} \nabla^{j} \Delta_{t} \phi \\
& \approx{ }^{i} \nabla^{j} \Delta_{t} N \lambda
\end{aligned}
$$

Referring to a previous study, the clock and atmosphererelated terms are neglected after the differencing process. ${ }^{15,16)}$ In addition, the carrier-phase noise term is also negligible because the standard deviation of the GPS L1 carrier-phase measurement is conservatively about $3 \mathrm{~mm}$. Then, only the cycle slip term remains after the time and satellite difference.

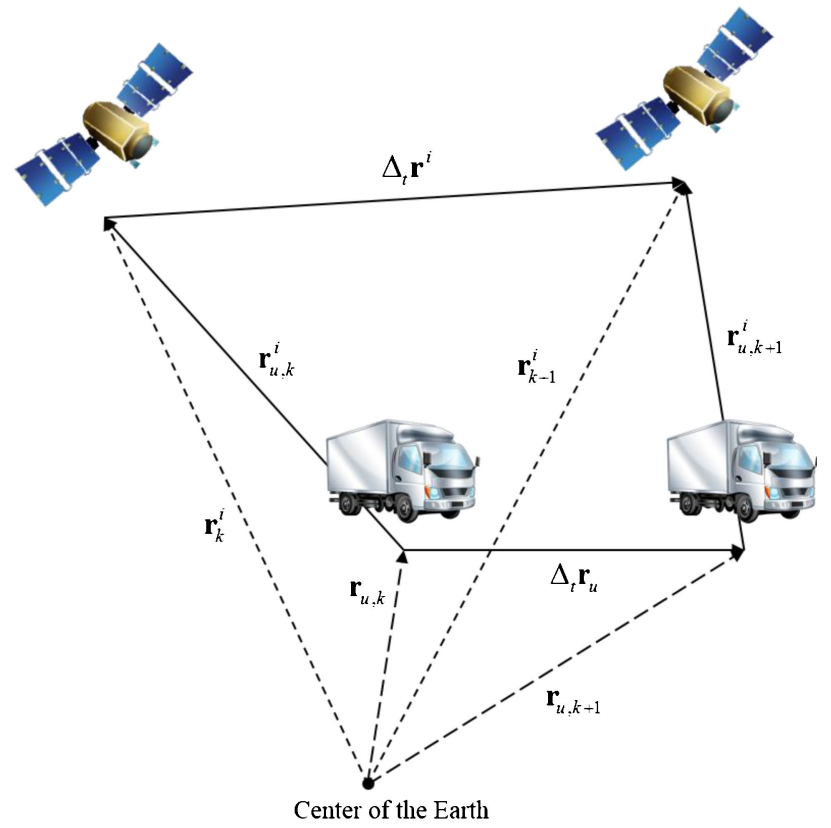

Fig. 1. User and satellite configuration during consecutive GPS epochs.
Next, the error of the predicted carrier phase from the INS is investigated.

$$
\delta^{i} \nabla^{j} \Delta_{t} \phi_{\mathrm{INS}}={ }^{i} \nabla^{j} \Delta_{t} \bar{\phi}_{\mathrm{INS}}-{ }^{i} \nabla^{j} \Delta_{t} \phi
$$

The estimated carrier phase in Eq. (5) can be expressed as follows.

$$
\begin{aligned}
{ }^{i} \nabla^{j} \Delta_{t} \bar{\phi}_{\mathrm{INS}}= & \left(\overline{\boldsymbol{r}}_{k+1}^{i}-\overline{\boldsymbol{r}}_{u, k+1}\right) \cdot \overline{\boldsymbol{e}}_{k+1}^{i}-\left(\overline{\boldsymbol{r}}_{k}^{i}-\overline{\boldsymbol{r}}_{u, k}\right) \cdot \overline{\boldsymbol{e}}_{k}^{i} \\
& -\left(\overline{\boldsymbol{r}}_{k+1}^{j}-\overline{\boldsymbol{r}}_{u, k+1}\right) \cdot \overline{\boldsymbol{e}}_{k+1}^{j}+\left(\overline{\boldsymbol{r}}_{k}^{j}-\overline{\boldsymbol{r}}_{u, k}\right) \cdot \overline{\boldsymbol{e}}_{k}^{j}
\end{aligned}
$$

Before further derivation, we use the following assumption for the line-of-sight vector. According to a previous study, ${ }^{17)}$ the angle between two successive line-of-sight vectors is the order of $10^{-4}$ radian.

$$
\begin{aligned}
\overline{\boldsymbol{e}}_{k+1}^{i} & \approx \boldsymbol{e}_{k+1}^{i} \\
\boldsymbol{e}_{k+1}^{i} & \approx \boldsymbol{e}_{k}^{i}
\end{aligned}
$$

Additionally, we assume that the relative satellite position between the GPS data epochs can be calculated from ephemeris data with sufficient accuracy.

$$
\Delta_{t} \overline{\boldsymbol{r}}_{k+1}^{i} \approx \Delta_{t} \boldsymbol{r}_{k+1}^{i}
$$

This is verified using the broadcasted ephemeris data and precise orbit data from the international GNSS service (IGS) final product as shown in Figs. 2 and 3.

In the figures, the consecutive GPS data epoch interval is $1 \mathrm{sec}$. It is also noticed that the relative satellite position error between the GPS data epochs is small, as shown in Fig. 3.

Then, Eq. (6) is modified as follows.

$$
{ }^{i} \nabla^{j} \Delta_{t} \bar{\phi}_{\mathrm{INS}}=\Delta_{t} \boldsymbol{r}^{i} \cdot \boldsymbol{e}_{k}^{i}-\Delta_{t} \boldsymbol{r}^{j} \cdot \boldsymbol{e}_{k}^{j}-\Delta_{t} \overline{\boldsymbol{r}}_{u} \cdot{ }^{i} \nabla^{j} \boldsymbol{e}_{k}
$$

In Eq. (9), it is noted that only the user position has considerable error, as follows.

$$
\Delta_{t} \overline{\boldsymbol{r}}_{u}=\Delta_{t} \boldsymbol{r}_{u}+\delta \Delta_{t} \boldsymbol{r}_{u}
$$

By substituting Eq. (10) into Eq. (9), Eq. (5) can be alternatively expressed as Eq. (11).

$$
\delta^{i} \nabla^{j} \Delta_{t} \phi_{\mathrm{INS}}=-\delta \Delta_{t} \boldsymbol{r}_{u} \cdot{ }^{i} \nabla^{j} \boldsymbol{e}_{k}
$$

Finally, the monitoring value equation can be represented as Eq. (12). During the derivation, we use several assump-

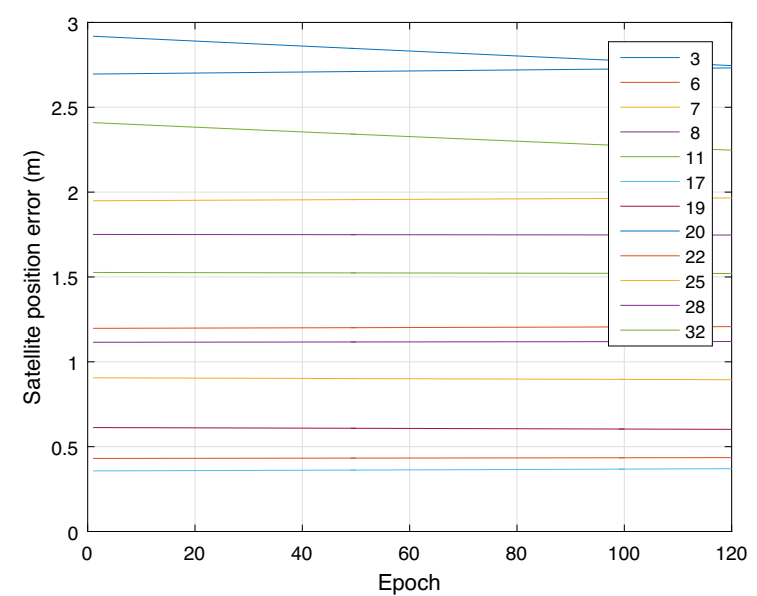

Fig. 2. Satellite position error from ephemeris data error. 


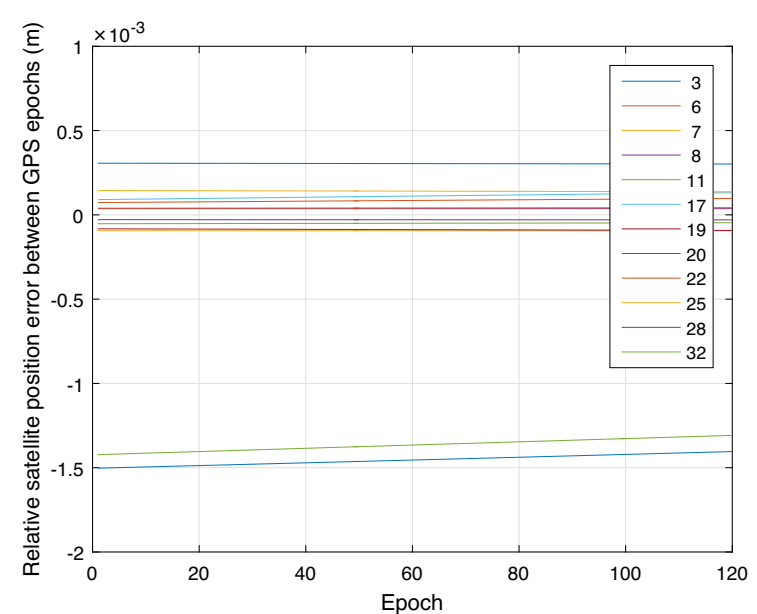

Fig. 3. Relative satellite position error between consecutive GPS epochs.

tions in Eqs. (4), (7), and (8). These assumptions are verified through static experiments, and the results are presented in Section 5.1.

$$
\bar{M}_{k+1}^{i}={ }^{i} \nabla^{j} \Delta_{t} N \lambda+\delta \Delta_{t} \boldsymbol{r}_{u} \cdot{ }^{i} \nabla^{j} \boldsymbol{e}_{k}
$$

The term $\delta \Delta_{t} \boldsymbol{r}_{u}$ represents the position estimation error between the consecutive GPS data epochs in Fig. 1, and its magnitude determines the cycle slip detection performance. If the position estimation error term is sufficiently small, we can detect the cycle slip precisely. However, if the position error is large, we cannot distinguish the cycle slip from the monitoring value because the cycle slip is concealed by the position error. Considering the ideal case, the monitoring value represents the amount of cycle slip.

Then, we define the monitoring value error as Eq. (13).

$$
\delta M_{k+1}^{i} \approx \delta \Delta_{t} \boldsymbol{r}_{u} \cdot{ }^{i} \nabla^{j} \boldsymbol{e}_{k}
$$

From Eq. (13), it is noted that the monitoring value error is mainly affected by the relative position estimation error between the epochs, not the absolute position error.

Therefore, it is suitable to use INS to estimate the relative position between the GPS data epochs. This is because the INS has good positioning accuracy when the integration time interval is short. In this case, the integration time interval is less than $1 \mathrm{sec}$.

From Eq. (13), we can define the upper bound of the monitoring value error as Eq. (14).

$$
\left|\delta M_{k+1}^{i}\right|_{\max } \leq\left.\left.\left|\delta \Delta_{t} \boldsymbol{r}_{u}\right|_{\max }\right|^{i} \nabla^{j} \boldsymbol{e}_{k}\right|_{\max }
$$

In Eq. (14), the maximum value of the SD line-of-sight vectors is approximated using $24 \mathrm{hr}$ satellite constellation simulation as shown in Figs. 4 and 5. The satellite position is calculated based on the broadcasted ephemeris data. As a result, the maximum value of SD line-of-sight vectors is smaller than 1.8 .

Then, Eq. (14) is simplified as follows.

$$
\left|\delta M_{k+1}^{i}\right|_{\max } \leq 1.8\left|\delta \Delta_{t} \boldsymbol{r}_{u}\right|_{\max }
$$

Finally, in order to satisfy the required cycle slip detection performance, the relative position error should be bounded,

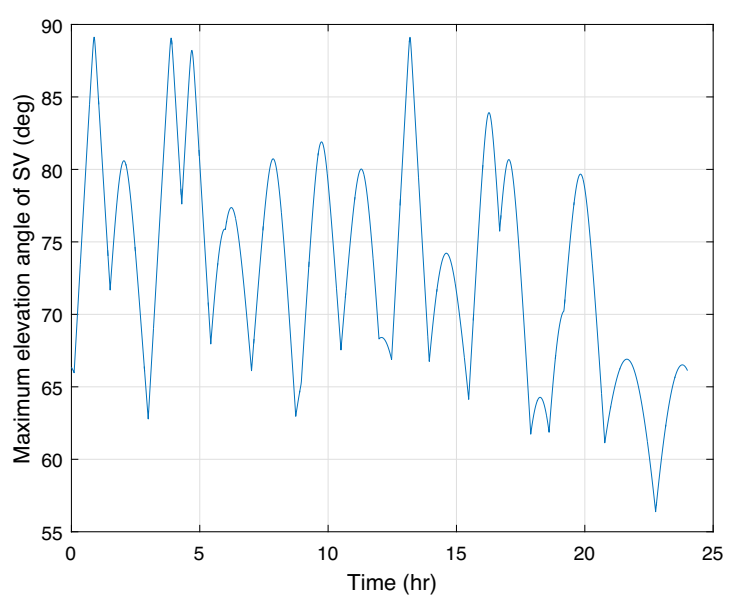

Fig. 4. Highest elevation angle of satellite (24hr).

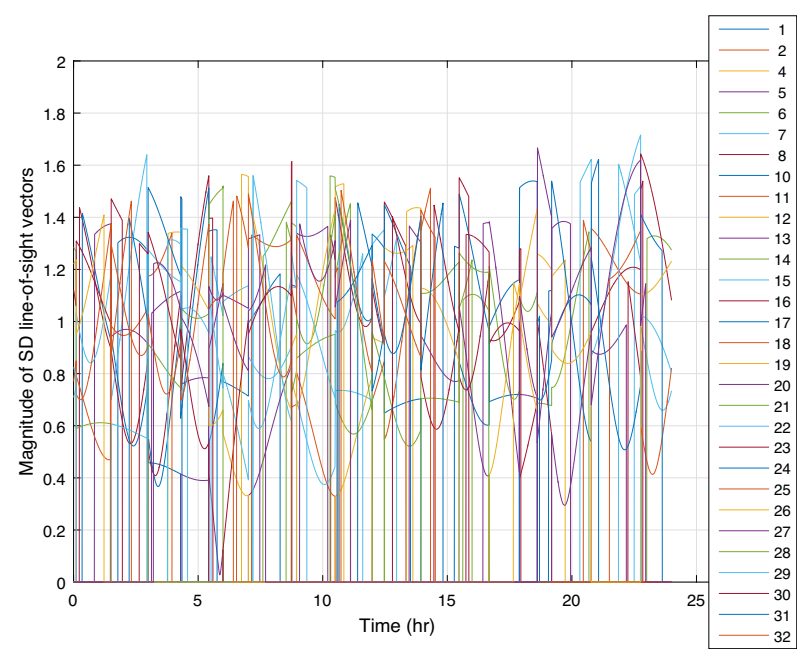

Fig. 5. Magnitude of SD line-of-sight vectors (24hr).

as in Eq. (16).

$$
\left|\delta \Delta_{t} \boldsymbol{r}_{u}\right|_{\max }<\frac{\text { threshold }}{1.8}
$$

\section{Relative Positioning Error Analysis}

\subsection{Inertial sensor error model}

In this proposed algorithm, the accelerometer and gyro are used to estimate the user position by integrating the outputs from the previous user position. However, the outputs of these two sensors contain errors, such as bias and noise, as well as the true values. In order to select the proper inertial sensor for the required position estimation accuracy, the errors of the INS must be investigated, and appropriate error models for these two sensors must be applied.

The error of the three-axis inertial sensor can be modeled as follows. ${ }^{18)}$

$$
\begin{aligned}
& \hat{\boldsymbol{y}}_{k}=\boldsymbol{y}_{k}+\boldsymbol{b}_{\mathrm{total}, k}+\boldsymbol{w}_{k} \\
& \boldsymbol{b}_{\mathrm{total}, k}=\boldsymbol{b}_{0}+\boldsymbol{b}_{k}
\end{aligned}
$$

The bias error can be separated into a constant component and a time-varying component. ${ }^{19)}$ The term $\boldsymbol{b}_{0}$ represents a 
constant null-shift. Its value is sometimes included in data sheets for inertial sensors and is referred to as the "turn-on to turn-off" bias. Additionally, the time-varying term represents the random bias drift or the residual of the bias after calibration.

The $\boldsymbol{b}_{0}$ is relatively easy to determine. ${ }^{20)}$ Moreover, the $\boldsymbol{b}_{0}$ is estimated using GPS/INS integrated navigation. Because the RTK has centimeter-level positioning accuracy, the $\boldsymbol{b}_{0}$ can be estimated well using the RTK position measurement if the vehicle maneuvers properly. After the filter convergence, $\boldsymbol{b}_{0}$ can be eliminated. Therefore, we focus on the bias drift term for relative position estimation error analysis.

Accordingly, a simplified error model for the inertial sensor can be expressed as follows.

$$
\hat{\boldsymbol{y}}_{k}=\boldsymbol{y}_{k}+\boldsymbol{b}_{k}+\boldsymbol{w}_{k}
$$

Using the simplified error model in Eq. (18), the gyro and accelerometer error model can be expressed as Eq. (19).

$$
\begin{aligned}
& \hat{\boldsymbol{f}}_{k}=\boldsymbol{f}_{k}+\boldsymbol{b}_{a, k}+\boldsymbol{w}_{a . k} \\
& \hat{\boldsymbol{\omega}}_{k}=\boldsymbol{\omega}_{k}+\boldsymbol{b}_{g, k}+\boldsymbol{w}_{g, k}
\end{aligned}
$$

The bias fluctuation can be simplified as a constant bias in a short time interval (i.e., within $1 \mathrm{sec}$ ). The amount of bias fluctuation is represented as the bias stability of an inertial sensor, which represents one-sigma value of the bias fluctuation.

$$
\begin{aligned}
\operatorname{var}\left(\boldsymbol{w}_{a, k}\right) & =\sigma_{a}^{2} I_{3 \times 3} \\
\operatorname{var}\left(\boldsymbol{w}_{g, k}\right) & =\sigma_{g}^{2} I_{3 \times 3} \\
\operatorname{var}\left(\boldsymbol{b}_{a, k}\right) & =\sigma_{b a}^{2} I_{3 \times 3} \\
\operatorname{var}\left(\boldsymbol{b}_{g, k}\right) & =\sigma_{b g}^{2} I_{3 \times 3}
\end{aligned}
$$

\subsection{INS propagation error analysis}

In Section 2.2, it was reported that the cycle slip detection performance highly depends on the position estimation error. In addition, in Section 3.1, the inertial sensor error was modeled. In this section, we analyze the INS error propagation due to the inertial sensor error. The results show that there is a connection between the INS position estimation accuracy and inertial sensor error.

The INS error propagation equations mechanized in an Earth-centered, Earth-fixed (ECEF) coordinate frame are as follows. Equation (21) represents the discrete time model of the INS error propagation equation. Because we consider the situation after $\boldsymbol{b}_{0}$ convergence, the constant bias of the inertial sensor is excluded from the state variable for simplicity.

$$
\begin{aligned}
& \boldsymbol{x}_{i+1}=F_{d} \boldsymbol{x}_{i}+G_{d} \boldsymbol{w}_{i} \\
& \boldsymbol{x}=\left[\begin{array}{lll}
\delta \boldsymbol{r}_{u} & \delta \boldsymbol{v}_{u} & \delta \boldsymbol{\psi}_{u}
\end{array}\right]^{\mathrm{T}} \\
& \boldsymbol{w}=\left[\begin{array}{ll}
\delta \boldsymbol{f} & \delta \boldsymbol{\omega}
\end{array}\right]^{\mathrm{T}}
\end{aligned}
$$

The components of the matrices in Eq. (21) are induced from the following continuous time model. ${ }^{21)}$ The Earth's rotation rate and gravity gradient are neglected in the system matrix $F$ because their magnitudes are very small compared to the other terms. The Earth's rotation rate is the order of $10^{-5}$ and gravity gradient is about $10^{-6}$.

$$
\begin{aligned}
F & =\left[\begin{array}{ccc}
0 & I & 0 \\
0 & 0 & -\left[C_{B}^{\bar{E}} \bar{f}\right]^{\times} \\
0 & 0 & 0
\end{array}\right] \\
G & =\left[\begin{array}{cc}
0 & 0 \\
C_{B}^{\bar{E}} & 0 \\
0 & C_{B}^{\bar{E}}
\end{array}\right]
\end{aligned}
$$

We assume that the numerical integration is performed using rectangle rule and the sampling time of an inertial sensor is sufficiently high. Then, the discrete model can be derived as follows from Eq. (22).

$$
\begin{aligned}
F_{d} & =e^{F T}=I+F T+\frac{1}{2 !} F^{2} T^{2} \\
G_{d} & =\left(\int_{0}^{T} e^{F \tau} \mathrm{d} \tau\right) G \\
& =\left(T I+\frac{T^{2}}{2 !} F+\frac{T^{3}}{3 !} F^{2}\right) G \\
F^{3} & =F^{4}=\cdots=0
\end{aligned}
$$

Each component of Eq. (23) is shown below.

$$
\begin{gathered}
F_{d}=\left[\begin{array}{ccc}
I & I T & -\frac{1}{2}\left[C_{B}^{\bar{E}} \overline{\boldsymbol{f}}\right]^{\times} T^{2} \\
0 & I & -\left[C_{B}^{\bar{E}} \overline{\boldsymbol{f}}\right]^{\times} T \\
0 & 0 & I
\end{array}\right] \\
G_{d}=\left[\begin{array}{cc}
\frac{1}{2} T^{2} C_{B}^{\bar{E}} & -\frac{1}{6}\left[C_{B}^{\bar{E}} \overline{\boldsymbol{f}}\right]^{\times} C_{B}^{\bar{E}} T^{3} \\
T C_{B}^{\bar{E}} & -\frac{1}{2}\left[C_{B}^{\bar{E}} \overline{\boldsymbol{f}}\right]^{\times} C_{B}^{\bar{E}} T^{2} \\
0 & C_{B}^{\bar{E}} T
\end{array}\right]
\end{gathered}
$$

From Eqs. (24) and (25), the error propagation after the measurement update for one INS epoch is derived as follows. For simplicity of analysis, we assume that the initial attitude of the vehicle coincides with the ECEF frame. This means the situation in which the vehicle is at the North Pole and the front of the vehicle is oriented toward the $x$-axis of the ECEF frame. In addition, we assume that the vehicle moves in the forward direction, and that during a short time interval (i.e., $1 \mathrm{sec}$ ), the attitude of the vehicle remains almost constant. This is illustrated in Fig. 6.

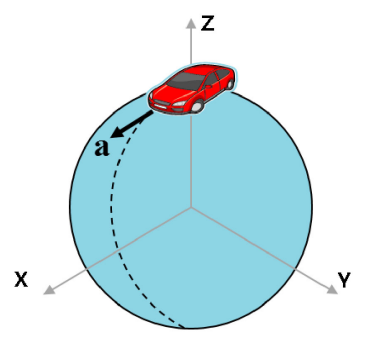

Fig. 6. Initial position and attitude of vehicle. 
Then, the direction cosine matrix in Eqs. (24) and (25) is simplified as an identity matrix. Accordingly, the INS propagation error equation can be rewritten as Eq. (26).

$$
\begin{aligned}
\delta \boldsymbol{r}_{u, i+1}^{-}= & \delta \boldsymbol{r}_{u, i}^{+}+T \delta \boldsymbol{v}_{u, i}^{+}-\frac{1}{2} T^{2}[\overline{\boldsymbol{f}}]^{\times} \delta \boldsymbol{\psi}_{u, i}^{+} \\
& +\frac{1}{2} T^{2}\left(\boldsymbol{b}_{a, i}+\boldsymbol{w}_{a, i}\right)-\frac{1}{6} T^{3}[\overline{\boldsymbol{f}}]^{\times}\left(\boldsymbol{b}_{g, i}+\boldsymbol{w}_{g, i}\right) \\
\delta \boldsymbol{v}_{u, i+1}^{-}= & \delta \boldsymbol{v}_{u, i}^{+}-T[\overline{\boldsymbol{f}}]^{\times} \delta \boldsymbol{\psi}_{u, i}^{+} \\
& +T\left(\boldsymbol{b}_{a, i}+\boldsymbol{w}_{a, i}\right)-\frac{1}{2} T^{2}[\overline{\boldsymbol{f}}]^{\times}\left(\boldsymbol{b}_{g, i}+\boldsymbol{w}_{g, i}\right) \\
\delta \boldsymbol{\psi}_{u, i+1}^{-}= & \delta \boldsymbol{\psi}_{u, i}^{+}+T\left(\boldsymbol{b}_{g, i}+\boldsymbol{w}_{g, i}\right)
\end{aligned}
$$

However, in general, the INS output rate is much higher than that of the GPS. The INS output rate is the same as that of the inertial sensor. Hence, we must propagate the INS for $n$ epochs in order to estimate the relative position between the GPS data epochs. Therefore, the propagation equation is extended for $n$ epochs, as in Eq. (27). The value of $n T$ is the same as the time interval between the GPS data epochs. For simplicity, the bias fluctuation is simplified as a constant bias within the GPS output time interval.

$$
\begin{aligned}
\delta \boldsymbol{r}_{u, i+n}^{-}= & \delta \boldsymbol{r}_{u, i}^{+}+n \boldsymbol{T} \delta \boldsymbol{v}_{u, i}^{+}-\frac{1}{2} n^{2} T^{2}[\overline{\boldsymbol{f}}]^{\times} \delta \boldsymbol{\psi}_{u, i}^{+} \\
& +\frac{1}{2} n^{2} T^{2} \boldsymbol{b}_{a, i}-\frac{1}{6} n^{3} T^{3}[\overline{\boldsymbol{f}}]^{\times} \boldsymbol{b}_{g, i} \\
& +\frac{1}{2} T^{2} \sum_{j=i}^{n+i-1} \boldsymbol{w}_{a, j}+T^{2} \sum_{k=i}^{n+i-1} \sum_{j=i}^{k-1} \boldsymbol{w}_{a, j} \\
& -\frac{1}{6} T^{3}[\overline{\boldsymbol{f}}]^{\times} \sum_{j=i}^{n+i-1} \boldsymbol{w}_{g, j}-T^{3}[\overline{\boldsymbol{f}}]^{\times} \sum_{k=i}^{n+i-1} \sum_{j=i}^{k-1} \boldsymbol{w}_{g, j} \\
& -T^{3}[\overline{\boldsymbol{f}}]^{\times} \sum_{l=i}^{n+i-1} \sum_{k=i}^{l-1} \sum_{j=i}^{k-1} \boldsymbol{w}_{g, j}
\end{aligned}
$$

In Eq. (27), the states at the $i$ epoch represent the results of the GPS measurement update. Moreover, the states at the $i+n$ epoch represent the INS propagation results before the GPS measurement update at that epoch. Thus, the position estimation error in Eq. (16) is expressed as follows. This is illustrated in Fig. 7.

$$
\delta \Delta_{t} \boldsymbol{r}_{u}=\delta \boldsymbol{r}_{u, i+n}^{-}-\delta \boldsymbol{r}_{u, i}^{+}
$$

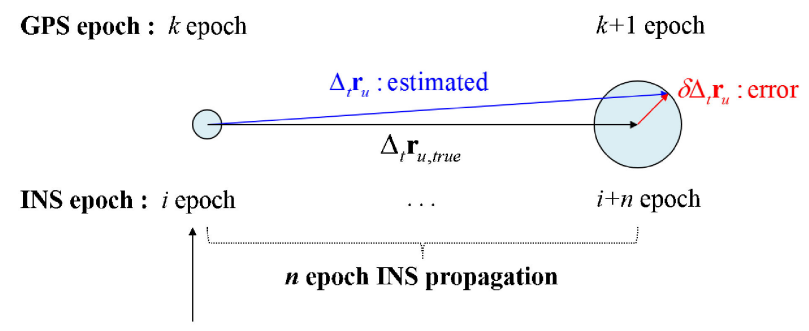

\section{GPS-RTK measurement update}

Fig. 7. Relative position estimation between consecutive GPS epochs.
From the results of Eqs. (27) and (28), it can be noted that relative position estimation accuracy depends on the velocity, attitude estimation error at the $i$ epoch, and inertial sensor error. From the modeling of the inertial sensor error in Section 3.1, $\boldsymbol{b}$ and $\boldsymbol{w}$ represent the bias stability and the Gaussian noise of the inertial sensor, respectively. If we predict the velocity and attitude estimation error at the $i$ epoch and the inertial sensor error influence, the relative position estimation error can be predicted according to the inertial sensor choice.

\subsection{Relative position error bounding}

The position estimation error can be bounded using Eq. (29).

$$
\begin{aligned}
\left|\delta \Delta_{t} \boldsymbol{r}_{u}\right|_{\max } \leq & \left|n T \delta \boldsymbol{v}_{u, i}^{+}\right|_{\max }+\left|\frac{1}{2} n^{2} T^{2}[\overline{\boldsymbol{f}}]^{\times} \delta \boldsymbol{\psi}_{u, i}^{+}\right|_{\max } \\
& +\left|\frac{1}{2} n^{2} T^{2} \boldsymbol{b}_{a, i}\right|_{\max }+\left|\frac{1}{6} n^{3} T^{3}[\overline{\boldsymbol{f}}]^{\times} \boldsymbol{b}_{a, i}\right|_{\max } \\
& +|\boldsymbol{A}|_{\max }+|\boldsymbol{B}|_{\max }
\end{aligned}
$$

In Eq. (29), the effects of the Gaussian noise error of the accelerometer and gyro are expressed individually as Eq. (30).

$$
\begin{aligned}
\boldsymbol{A}= & \frac{1}{2} T^{2} \sum_{j=i}^{n+i-1} \boldsymbol{w}_{a, j}+T^{2} \sum_{k=i}^{n+i-1} \sum_{j=i}^{k-1} \boldsymbol{w}_{a, j} \\
\boldsymbol{B}= & \frac{1}{6} T^{3}[\overline{\boldsymbol{f}}]^{\times} \sum_{j=i}^{n+i-1} \boldsymbol{w}_{g, j}+T^{3}[\overline{\boldsymbol{f}}]^{\times} \sum_{k=i}^{n+i-1} \sum_{j=i}^{k-1} \boldsymbol{w}_{g, j} \\
& +T^{3}[\overline{\boldsymbol{f}}]^{\times} \sum_{l=i}^{n+i-1} \sum_{k=i}^{l-1} \sum_{j=i}^{k-1} \boldsymbol{w}_{g, j}
\end{aligned}
$$

In Eq. (30), the bounds of the specific acceleration of the vehicle can be represented using the vehicle acceleration and gravity as follows. We assume that the vehicle accelerates at $a \mathrm{~m} / \mathrm{sec}^{2}$.

$$
|\overline{\boldsymbol{f}}|_{\max }=f=\sqrt{a^{2}+g_{0}^{2}}
$$

In the case of the attitude error, the error can be divided into the tilt angle error, $\delta \psi_{u x}$ and $\delta \psi_{u y}$, and the azimuth angle error, $\delta \psi_{u z}$, as in Eq. (32).

$$
\left|\delta \boldsymbol{\psi}_{u, i}^{+}\right|_{\max }=\left[\left|\delta \psi_{u x, i}^{+}\right|_{\max }\left|\delta \psi_{u y, i}^{+}\right|_{\max }\left|\delta \psi_{u z, i}^{+}\right|_{\max }\right]^{\mathrm{T}}
$$

Then, Eq. (29) can be rewritten as Eqs. (33) and (34).

$$
\begin{aligned}
\left|\delta \Delta_{t} \boldsymbol{r}_{u}\right|_{\max } \leq & n T\left|\delta \boldsymbol{v}_{u, i}^{+}\right|_{\max } \\
& +\frac{1}{2} n^{2} T^{2}\left|[\overline{\boldsymbol{f}}]^{\times} \delta \boldsymbol{\psi}_{u, i}^{+}\right|_{\max } \\
& +\frac{1}{2} n^{2} T^{2}\left|\boldsymbol{b}_{a, i}\right|_{\max }+\frac{1}{6} n^{3} T^{3} f\left|\boldsymbol{b}_{g, i}\right|_{\max } \\
& +|\boldsymbol{A}|_{\max }+|\boldsymbol{B}|_{\max } \\
\left|[\overline{\boldsymbol{f}}]^{\times} \delta \boldsymbol{\psi}_{u, i}^{+}\right|_{\max } \leq & g_{0}\left|\delta \psi_{u x, i}^{+}\right|_{\max }+\left(a+g_{0}\right)\left|\delta \psi_{u y, i}^{+}\right|_{\max }+a\left|\delta \psi_{u z, i}^{+}\right|
\end{aligned}
$$

In Eq. (33), the terms on the right side can be divided into two groups. The first group is the velocity and attitude estimation error after the measurement update. The second 
group is the errors caused by the inertial sensor bias drift and noise.

For the first group, the magnitude of the estimation error can be approximated from the covariance of the RTK/INS filter after a certain maneuver. We consider the two-step maneuver for filter convergence. First, the vehicle proceeds at a constant velocity and then accelerates in the forward direction. After the first maneuver, the velocity and tilt angle errors are converged. In addition, after the second maneuver, the azimuth angle error is converged. For simplicity, we consider the situation described in Fig. 6.

In the first maneuver, the horizontal and vertical channel of the RTK/INS filter are decoupled as follows. Equation (35) shows the horizontal channel and Eq. (36) represents the vertical channel. It is clear that the position, velocity, and tilt angle errors are observable from the RTK position measurement. However, the azimuth angle error is unobservable.

$$
\begin{aligned}
\delta \dot{r}_{u x} & =\delta v_{u x} \\
\delta \dot{v}_{u x} & =g_{0} \delta \psi_{u y}+w_{a x} \\
\delta \dot{\psi}_{u y} & =w_{g y} \\
\delta \dot{r}_{u y} & =\delta v_{u y} \\
\delta \dot{v}_{u y} & =-g_{0} \delta \psi_{u x}+w_{a y} \\
\delta \dot{\psi}_{u x} & =w_{g x} \\
& \delta \dot{r}_{u z}=\delta v_{u z} \\
\delta \dot{v}_{u z} & =w_{a z}
\end{aligned}
$$

During the first maneuver, the variance of the azimuth angle error remains almost constant from the initial value as follows. $P$ represents the $9-$ by- 9 covariance matrix of the filter.

$$
\operatorname{var}\left(\delta \psi_{u z}\right) \approx \sqrt{P_{99,0}}
$$

After the first maneuver, the position, velocity, and tilt angle errors are converged. Next, in the second maneuver, the velocity error equation of the $y$-axis in Eq. (35) is changed to Eq. (38) because of the forward acceleration $a$.

$$
\delta \dot{v}_{u y}=-g_{0} \delta \psi_{u x}+a \delta \psi_{u z}+w_{a y}
$$

At the beginning of the second maneuver, the variance of the attitude error in the $x$-axis and $z$-axis in Eq. (38) have the following relationship. After the first maneuver, the variance of the attitude error in the $x$-axis has been converged, while the variance of attitude in the $z$-axis remains the initial value.

$$
\operatorname{var}\left(\delta \psi_{u x}\right) \ll \operatorname{var}\left(\delta \psi_{u z}\right)
$$

In this case, according to the convergence theorem of a previous study, ${ }^{20)}$ the azimuth angle turns out to be observable and its variance is converged. It is verified through the simulation in Section 5.2.

This enables the covariance of the RTK/INS filter to be predicted using a simple simulation.

The covariance prediction process is shown in Table 1 .

The matrices in Table 1 are shown as in Eq. (40). In Eq. (40), the $\sigma_{\mathrm{RTK}}$ is $1 \mathrm{~cm}$ in general.

$$
\begin{aligned}
W & =\left[\begin{array}{cc}
\sigma_{a}^{2} I_{3 \times 3} & 0 \\
0 & \sigma_{g}^{2} I_{3 \times 3}
\end{array}\right] \\
R & =\sigma_{\mathrm{RTK}}^{2} I_{3 \times 3} \\
H & =\left[\begin{array}{ll}
I_{3 \times 3} & 0_{3 \times 6}
\end{array}\right]
\end{aligned}
$$

We express the resulting covariance matrix after proper maneuvering as $P$, and it is a 9-by-9 matrix. Only the diagonal elements of matrix $P$ are used to predict the errors of the first group. Additionally, the tilt angle error is also affected by the accelerometer bias estimation error. ${ }^{20,21)}$ This implies that the gravity influence caused by the tilt angle error has a similar magnitude to the accelerometer bias.

Next, the errors of the first group can be predicted as follows.

Equation (41) represents the velocity error. We take the two-sigma value for the maximum bound. That is, we assume that $95 \%$ of the error is included within the bounds.

$$
\begin{aligned}
\left|\delta \boldsymbol{v}_{u, i}^{+}\right|_{\max } & =\sqrt{\left|\delta v_{u x, i}^{+}\right|_{\max }^{2}+\left|\delta v_{u y, i}^{+}\right|_{\max }^{2}+\left|\delta v_{u z, i}^{+}\right|_{\max }^{2}} \\
\left|\delta v_{u x, i}^{+}\right|_{\max } & \approx 2 \sqrt{P_{44}} \\
\left|\delta v_{u y, i}^{+}\right|_{\max } & \approx 2 \sqrt{P_{55}} \\
\left|\delta v_{u z, i}^{+}\right|_{\max } & \approx 2 \sqrt{P_{66}}
\end{aligned}
$$

Equation (42) represents the attitude error. As mentioned before, the tilt angle errors are also affected by the accelerometer bias estimation error, as shown in Eq. (42).

$$
\begin{aligned}
\left|\delta \psi_{u x, i}^{+}\right|_{\max } & \approx \sqrt{\left(\frac{\left|b_{a y, i}\right|_{\max }}{g_{0}}\right)^{2}+\left(2 \sqrt{P_{77}}\right)^{2}} \\
\left|\delta \psi_{u y, i}^{+}\right|_{\max } & \approx \sqrt{\left(\frac{\left|b_{a x, i}\right|_{\max }}{g_{0}}\right)^{2}+\left(2 \sqrt{P_{88}}\right)^{2}} \\
\left|\delta \psi_{u z, i}^{+}\right|_{\max } & \approx 2 \sqrt{P_{99}}
\end{aligned}
$$

For the analysis of the second group, the magnitude of the three-dimensional error vector is expressed using the singledimensional error, as in Eqs. (43) and (44), because for manufacturing the IMU, the inertial sensor for each axis generally has the same specifications.

$$
\begin{aligned}
\left|\boldsymbol{b}_{a, i}\right|_{\text {max }} & =\sqrt{\left|b_{a x, i}\right|_{\text {max }}^{2}+\left|b_{a y, i}\right|_{\text {max }}^{2}+\left|b_{a z, i}\right|_{\text {max }}^{2}} \\
\left|\boldsymbol{b}_{g, i}\right|_{\text {max }} & =\sqrt{\left|b_{g x, i}\right|_{\text {max }}^{2}+\left|b_{g y, i}\right|_{\text {max }}^{2}+\left|b_{g z, i}\right|_{\text {max }}^{2}} \\
|\boldsymbol{A}|_{\text {max }} & =\sqrt{3}|A|_{\text {max }} \\
|\boldsymbol{B}|_{\text {max }} & =\sqrt{3}|B|_{\text {max }}
\end{aligned}
$$

Table 1. Covariance calculation of RTK/INS filter after maneuvering.

\begin{tabular}{ll}
\hline Initialization & $P_{i}^{-}=P_{0}$ \\
\hline Covariance propagation (INS epoch) & $P_{i+1}^{-}=F_{d} P_{i}^{-} F_{d}^{\mathrm{T}}+G_{d} W G_{d}^{\mathrm{T}}$ \\
\hline Covariance innovation (GPS epoch) & $K_{i+1}=P_{i+1}^{-} H^{\mathrm{T}}\left(H P_{i+1}^{-} H^{\mathrm{T}}+R\right)^{-1}$ \\
& $P_{i+1}^{+}=P_{i+1}^{-}-K_{i+1} H P_{i+1}^{-}$ \\
\hline
\end{tabular}


In addition, for the bounds of the inertial sensor bias drift, we use the two-sigma (95\%) value of the bias stability, which is generally specified in the inertial sensor data sheet.

$$
\begin{aligned}
& \left|b_{a x, i}\right|_{\text {max }}=\left|b_{a y, i}\right|_{\text {max }}=\left|b_{a z, i}\right|_{\text {max }}=2 \sigma_{b a} \\
& \left|b_{g x, i}\right|_{\text {max }}=\left|b_{g y, i}\right|_{\text {max }}=\left|b_{g z, i}\right|_{\text {max }}=2 \sigma_{b g}
\end{aligned}
$$

In Eq. (43), the effects of the Gaussian error for a single axis can be written as below.

$$
\begin{aligned}
A= & \frac{1}{2} T^{2} \sum_{j=i}^{n+i-1} w_{a, j}+T^{2} \sum_{k=i}^{n+i-1} \sum_{j=i}^{k-1} w_{a, j} \\
B= & \frac{1}{6} T^{3} f \sum_{j=i}^{n+i-1} w_{g, j}+T^{3} f \sum_{k=i}^{n+i-1} \sum_{j=i}^{k-1} w_{g, j} \\
& +T^{3} f \sum_{l=i}^{n+i-1} \sum_{k=i}^{l-1} \sum_{j=i}^{k-1} w_{g, j}
\end{aligned}
$$

The variance of Eq. (45) can be calculated as in Eq. (46).

$$
\begin{aligned}
& \operatorname{var}(A)=\frac{4 n^{3}-n}{12} T^{4} \sigma_{a}^{2} \\
& \operatorname{var}(B)=\frac{9 n^{5}-5 n^{3}+n}{180} f^{2} T^{6} \sigma_{g}^{2}
\end{aligned}
$$

Moreover, we define the bounds of each value using the two-sigma (95\%) value of noise standard deviation as shown below.

$$
\begin{aligned}
|A|_{\text {max }} & =2 \sqrt{\operatorname{var}(A)} \\
|B|_{\text {max }} & =2 \sqrt{\operatorname{var}(B)}
\end{aligned}
$$

Finally, all of the errors in estimating the relative position are analyzed. In addition, the relative position estimation error is bounded using the inertial sensor specifications, as in Eq. (48). This means that by using Eq. (48), we can select the proper inertial sensor for the required cycle slip detection. We verify the derivation results term-by-term using the simulation data in Section 5.3.

$$
\left|\delta \Delta_{t} \boldsymbol{r}_{u}\right|_{\max } \leq f n\left(P, \sigma_{b a}, \sigma_{b g}, \sigma_{a}, \sigma_{g}, n, T, f\right)
$$

The details of Eq. (48) are shown as in Eq. (49).

$$
\begin{aligned}
& f n\left(P, \sigma_{b a}, \sigma_{b g}, \sigma_{a}, \sigma_{g}, n, T, f\right) \\
& =2 n T \sqrt{P_{44}+P_{55}+P_{66}} \\
& +\frac{1}{2} n^{2} T^{2}\left\{\begin{array}{c}
g_{0} \sqrt{\left(\frac{2 \sigma_{b a}}{g_{0}}\right)^{2}+\left(2 \sqrt{P_{77}}\right)^{2}} \\
+\left(a+g_{0}\right) \\
+2 a \sqrt{\left(\frac{2 \sigma_{b a}}{g_{0}}\right)^{2}+\left(2 \sqrt{P_{88}}\right)^{2}}
\end{array}\right\} \\
& +2 \sqrt{3}\left(n^{2} T^{2} \sigma_{b a}\right)+2 \sqrt{3}\left(\frac{1}{6} n^{3} T^{3} f \sigma_{b g}\right) \\
& +2 \sqrt{3} \sqrt{\frac{4 n^{3}-n}{12} T^{4} \sigma_{a}^{2}}+2 \sqrt{3} \sqrt{\frac{9 n^{5}-5 n^{3}+n}{180} T^{6} f^{2} \sigma_{g}^{2}}
\end{aligned}
$$

\section{Optimal Inertial Sensor Selection}

In Section 3.3, we derived the bounds of the relative position estimation error according to the inertial sensor specifications. Using this equation, the proper inertial sensor can be selected among the IMU candidates.

$$
f n\left(P, \sigma_{b a}, \sigma_{b g}, \sigma_{a}, \sigma_{g}, n, T, f\right)<\frac{\text { threshold }}{1.8}
$$

By selecting the upper limit of allowable inertial sensor errors from Eq. (50), we can minimize the cost of the inertial sensor, while satisfying the requirements. We give the following example for users.

We set the threshold $=0.13 \mathrm{~m}, n=100, T=0.01 \mathrm{sec}$, $a=0 \mathrm{~m} / \mathrm{sec}^{2}$ for one cycle slip detection. Then the relative positioning error should be smaller than $0.0722 \mathrm{~m}$ for satisfying Eq. (50).

To select the proper IMU, we create IMU candidates considering the different grades of commercial IMU, as shown in Table 2. For the user, the IMU candidates should be created among the interested commercial IMUs.

After selection of the IMU candidates, the variance of the velocity and attitude errors can be predicted using the process expressed in Table 1. Table 3 shows the resulting standard deviation after $25 \mathrm{sec}$ of driving at constant velocity and $25 \mathrm{sec}$ of acceleration in the forward direction at $1 \mathrm{~m} / \mathrm{sec}^{2}$.

Then, by calculating the relative positioning error of the IMU candidates using Eq. (49), the optimal sensor can be selected. Table 4 shows the relative positioning error of the IMU candidates. It is be noted that the CIMU2 is suitable.

As a result, one cycle slip of the carrier-phase measurement can be detected by the low-cost IMU. Moreover, this agrees with the motivation of inertial-aided cycle slip detection, which is to achieve high performance while requiring low cost compared with other methods using a dualfrequency receiver.

Table 2. IMU candidates.

\begin{tabular}{lcccc}
\hline \multicolumn{1}{c}{ Model } & $\begin{array}{c}\text { Gyro noise } \\
(\mathrm{deg} / \sqrt{\mathrm{h}})\end{array}$ & $\begin{array}{c}\text { Gyro bias } \\
\text { stability } \\
(\mathrm{deg} / \mathrm{h})\end{array}$ & $\begin{array}{c}\text { Acc. noise } \\
(\mathrm{m} / \mathrm{sec} / \sqrt{\mathrm{h}})\end{array}$ & $\begin{array}{c}\text { Acc. bias } \\
\text { stability } \\
(\mathrm{mg})\end{array}$ \\
\hline $\begin{array}{l}\text { CIMU1: } \\
\text { Tactical grade }\end{array}$ & 0.0667 & 1 & 0.033 & 0.25 \\
\hline $\begin{array}{l}\text { CIMU2: } \\
\text { MEMS grade }\end{array}$ & 2 & 25 & 0.2 & 0.2 \\
\hline
\end{tabular}

Table 3. Converged standard deviation.

\begin{tabular}{lllllll}
\hline Model & $\begin{array}{c}\sqrt{P_{44}} \\
\mathrm{~m} / \mathrm{sec}\end{array}$ & $\begin{array}{c}\sqrt{P_{55}} \\
\mathrm{~m} / \mathrm{sec}\end{array}$ & $\begin{array}{c}\sqrt{P_{66}} \\
\mathrm{~m} / \mathrm{sec}\end{array}$ & $\begin{array}{c}\sqrt{P_{77}} \\
\mathrm{deg}\end{array}$ & $\begin{array}{c}\sqrt{P_{88}} \\
\mathrm{deg}\end{array}$ & $\begin{array}{l}\sqrt{P_{99}} \\
\mathrm{deg}\end{array}$ \\
\hline CIMU1 & 0.002 & 0.002 & 0.001 & 0.005 & 0.003 & 0.04 \\
CIMU2 & 0.011 & 0.011 & 0.005 & 0.08 & 0.05 & 0.7 \\
\hline
\end{tabular}

Table 4. Relative positioning error of IMUs.

\begin{tabular}{cc}
\hline Model & Relative positioning error \\
\hline CIMU1 & $0.02 \mathrm{~m}$ \\
CIMU2 & $0.07 \mathrm{~m}$ \\
\hline
\end{tabular}




\section{Simulation Results}

\subsection{Static test for the validity of the assumptions}

To verify the validity of the assumptions that had been made during the monitoring value derivation, we conducted a static test. The assumptions, including regarding the satellite orbit error, clock errors, atmospheric related delays, and carrier phase noises, are negligible between two consecutive GPS epochs. In addition, the line-of-sight vectors between the GPS epochs are almost equal. We exclude ionospheric storm conditions. If an ionospheric storm occurrs, the assumptions cannot be preserved.

In the static test, we know the relative position vector between the epochs, the zero vector. Therefore, the only error sources of the monitoring value are the above-listed errors. By checking the magnitude of the monitoring value error from the static test, we can confirm the validity of the assumptions.

The static test was conducted in an open-sky environment. A Ublox LEA-6T and patch antenna were used for GPS raw data collection. The GPS receiver output rate was $1 \mathrm{~Hz}$. The stand-alone GPS solution was used as the user position. The SBAS corrections were disabled and we used the automotive mode for the receiver setting. Figure 8 is the sky plot of the static experiment. Figure 9 shows the monitoring value error of the static test. The numbers listed in the legend are the pseudorandom noise (PRN) numbers of the satellites. The PRN 5 satellite is used as the reference satellite.

The resulting magnitude of the monitoring value is at the millimeter-level. Therefore, in general conditions, the effects of the satellite orbit and clock errors, tropospheric and ionospheric delays, and carrier-phase noises on the monitoring value are negligible between two consecutive epochs. Additionally, the line-of-sight vectors between the two consecutive epochs are almost equal. These results verify the validity of the assumptions, which had been made during the monitoring value derivation process.

\subsection{Simulation results-RTK/INS filter covariance convergence}

In this section, to verify the covariance convergence of the filter, we simulate the maneuver shown in Fig. 10.

Figure 11 shows the resulting standard deviation of velocity and attitude errors of the simplified RTK/INS filer after the measurement update. The vertical line in the middle of the figure divides the maneuvers.

As derived in Section 3.3, the velocity and tilt angle errors are converged in the first maneuver and the azimuth angle error is converged in the second maneuver. The quantitative values are presented in Table 3.

\subsection{Simulation results-INS propagation error}

To verify the INS propagation error in Eq. (29), we conduct a simulation by inserting an error term-by-term. The simulation is conducted for the case of the example in Section 4. Additionally, the acceleration maneuver is also simulated, $a=2 \mathrm{~m} / \mathrm{sec}^{2}$.

All errors are assumed as zero-mean Gaussian. The bias errors are constant between the consecutive GPS epoch inter-

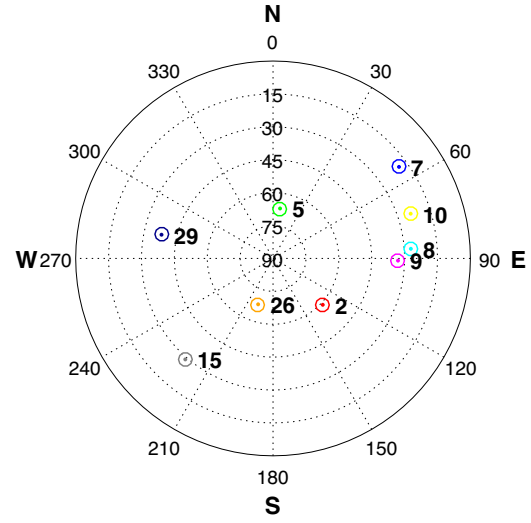

Fig. 8. Sky plot (static experiment).

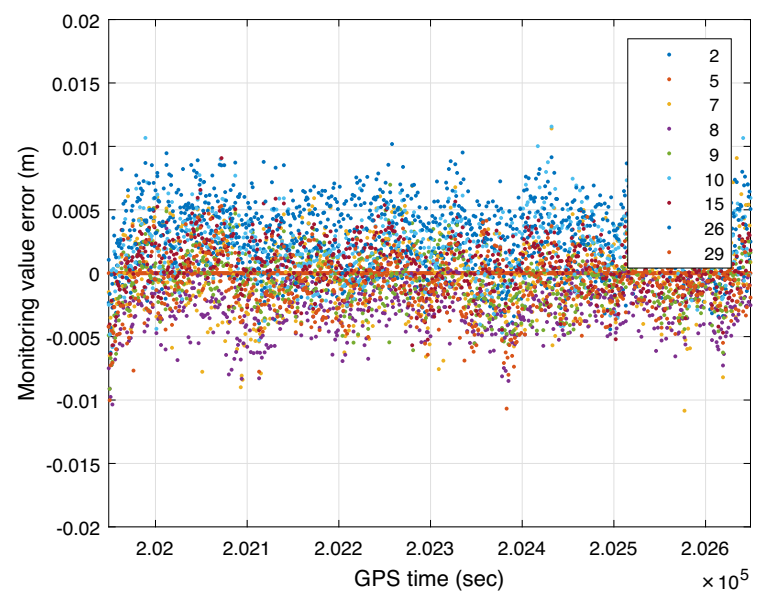

Fig. 9. Monitoring value error (static).

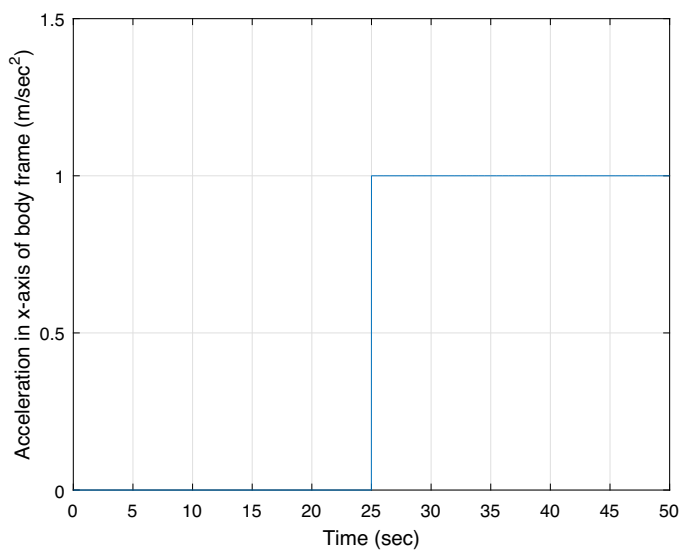

Fig. 10. Simulated maneuver (forward acceleration).

val, but change for each interval. In addition, the standard deviations of the velocity and attitude errors are created using the results of Table 3. The inertial sensor error is generated according to the MEMS IMU specification in Table 2. The inertial sensor output rate is set as $0.01 \mathrm{sec}$ and the GPS output rate is $1 \mathrm{sec}$.

Figures 12 and 13 show the relative position estimation error caused by the velocity error, attitude error, and inertial sensor error in the constant velocity and acceleration cases, respectively. Figure 14 shows the total relative position error 
Trans. Japan Soc. Aero. Space Sci., Vol. 59, No. 4, 2016
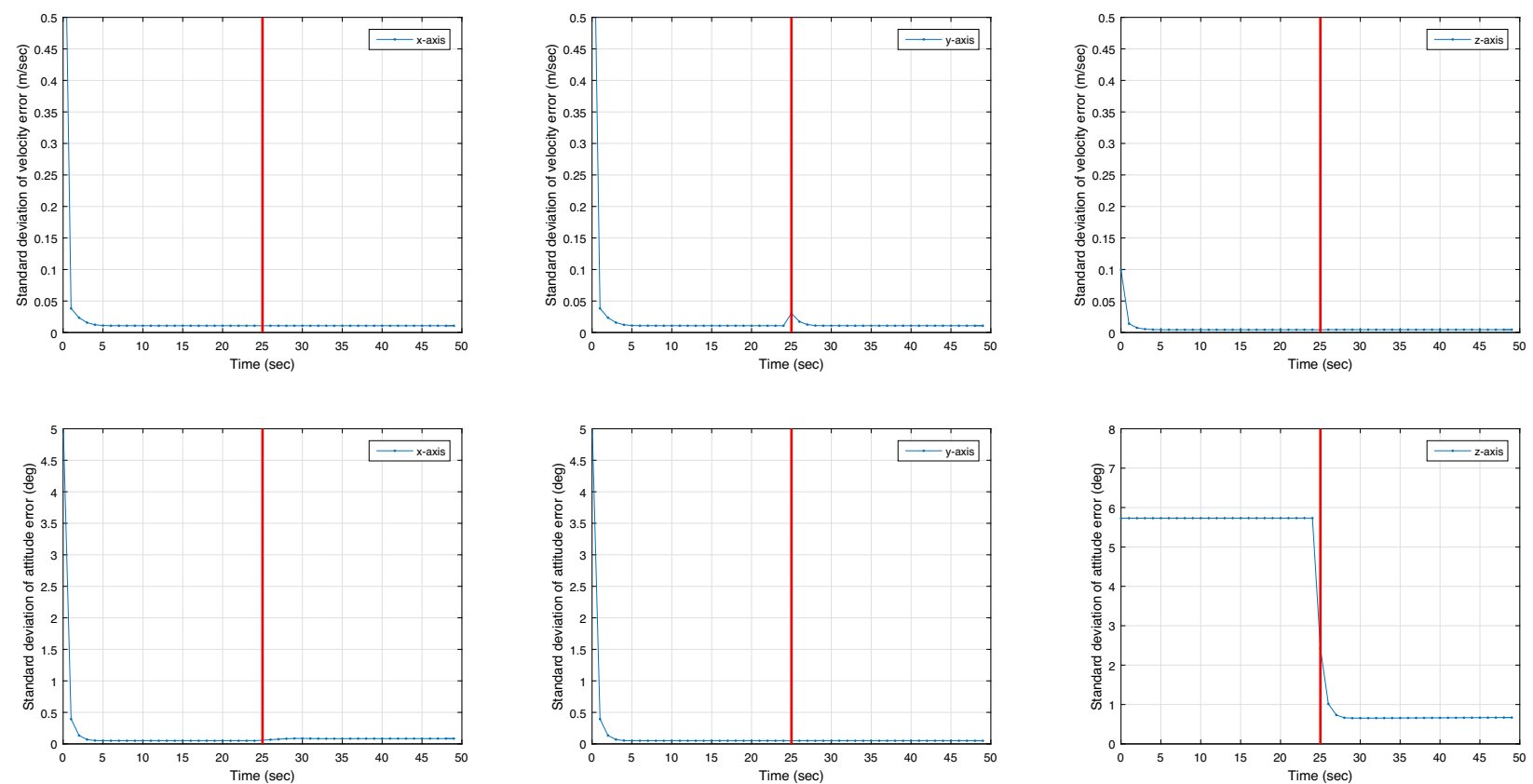

Fig. 11. Time history of standard deviation of RTK/INS filter during simulated maneuvering.
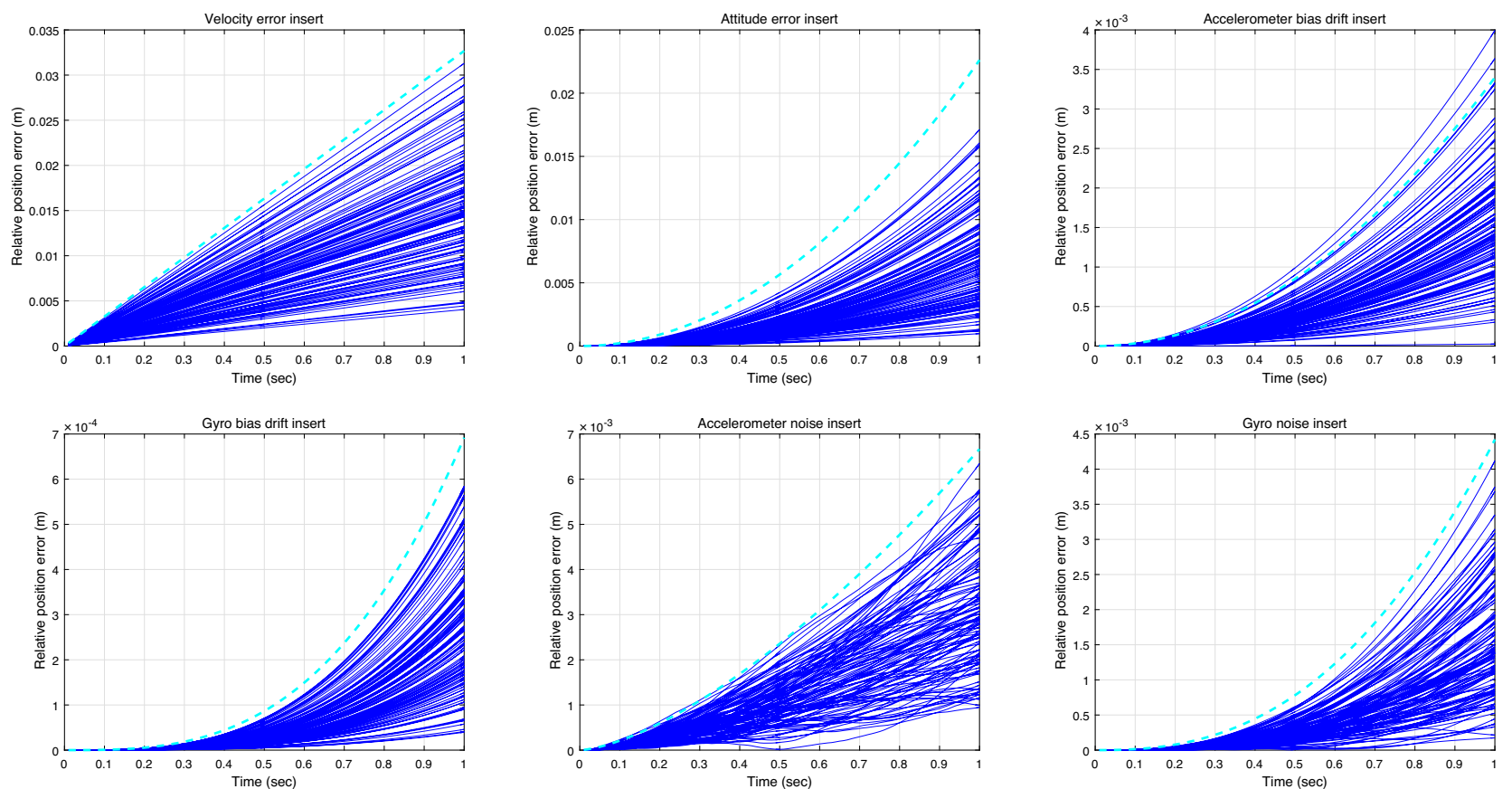

Fig. 12. Relative position estimation error (constant velocity case).

caused by the sum of the velocity, attitude, and inertial sensor errors in each case. In Figs. 12-14, the dashed lines are the theoretical bounds derived in the previous section, and the solid lines are the simulation results. A total of 120 epochs of GPS data are used.

In the constant velocity and acceleration cases, the velocity error is linearly propagated and predicted well by the theoretical results. The attitude error has a steeper slope than that of the velocity error and is also predicted well. In addition, the position error caused by the attitude error is much larger in the acceleration case than in the constant velocity case. The reason for this phenomenon is that the azimuth error is inserted in the position error multiplied by the horizontal acceleration. For the straight course at constant speed, the horizontal acceleration is zero, so that the azimuth angle error does not have much of an effect on the position error. However, in the case of acceleration, horizontal acceleration does exist and the azimuth angle error is much larger than the tilt angle error. Thus, even small horizontal acceleration causes considerable error in the position estimation error.

The inertial sensor error is also analyzed term-by-term. First, the accelerometer drift error causes error in the posi- 

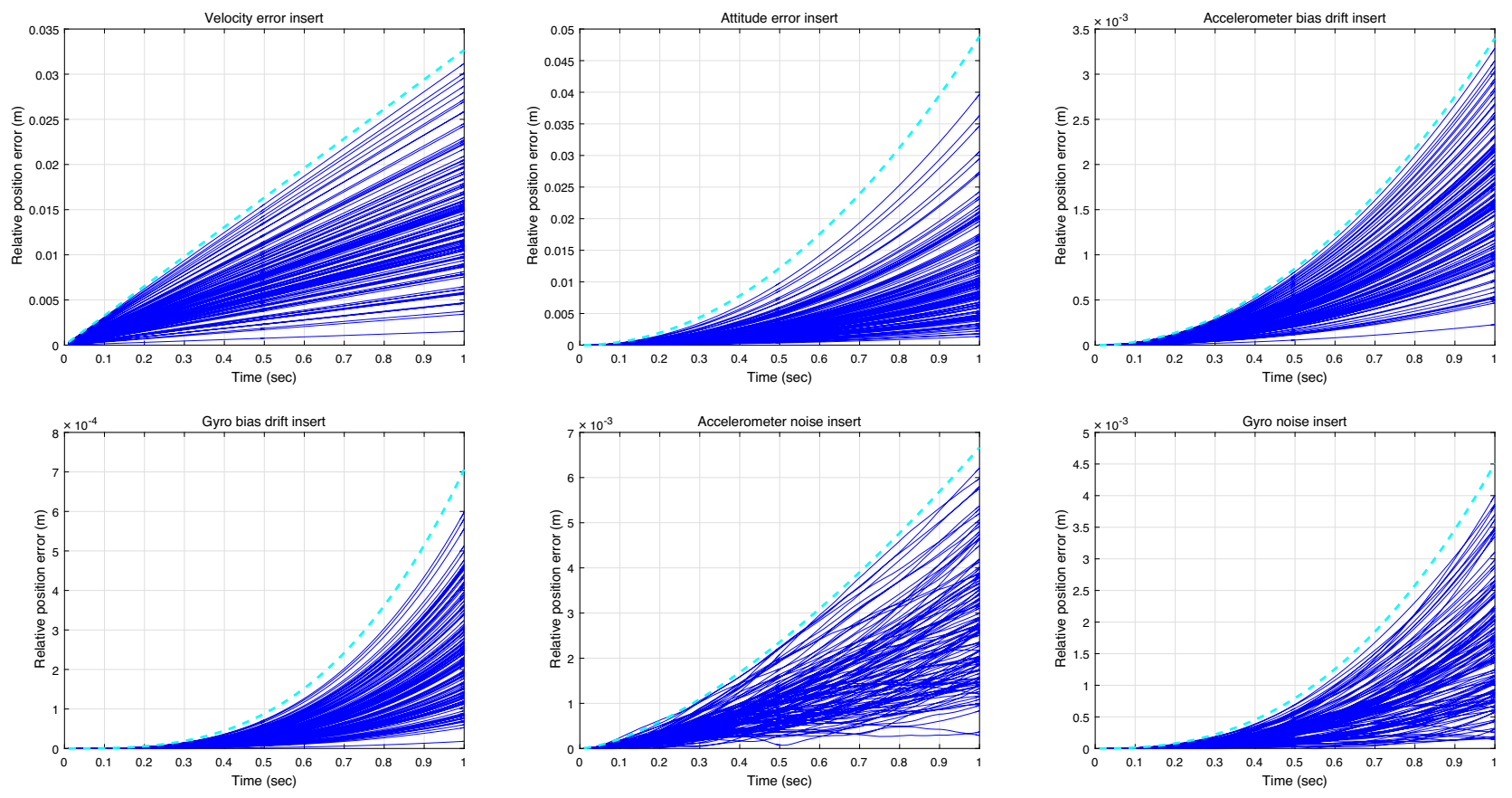

Fig. 13. Relative position estimation error (acceleration case).
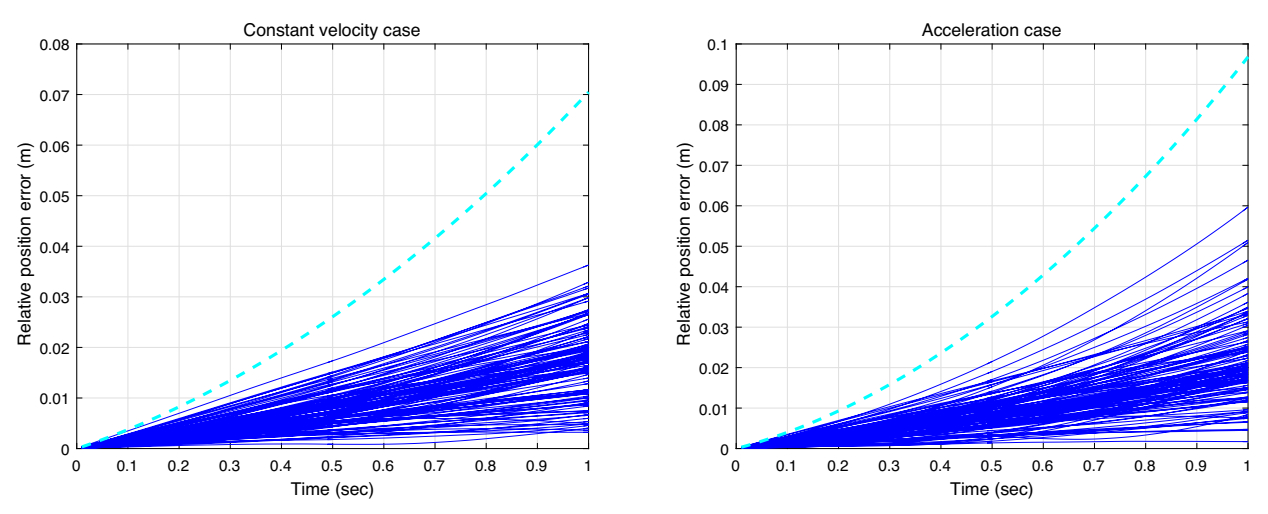

Fig. 14. Total relative position estimation error.

tion, which is a function of the propagated time to the power of two in both course cases. In addition, the gyro error increases the relative position error more rapidly than that of the accelerometer.

The simulation results are well bounded by the theoretical results.

Figure 14 shows the total position error due to the sum of the individual errors. Likewise, the theoretical deviation results matched the simulation results well in both the constant velocity and acceleration cases. The resulting total position error is larger in the acceleration case than in the constant velocity case because of the difference in the influence of the azimuth angle error.

\subsection{Simulation results—monitoring value error}

To verify the monitoring value error of the selected IMU, the GPS simulation data is generated regarding the errors in Table 5. Figure 15 shows the sky plot of the simulation data.

The relative position estimation error of the INS results in the error in the monitoring value multiplied by the line-ofsight vector.
Table 5. GPS simulation generation method.

\begin{tabular}{ll}
\hline \multicolumn{1}{c}{ GPS errors } & \multicolumn{1}{c}{ Generation strategy } \\
\hline Ephemeris error & $\begin{array}{l}\text { Difference between satellite positions based on } \\
\text { precise orbit and navigation data } \\
\text { Ionosphere map exchange format (IONEX) and } \\
\text { obliquity factor } \\
\text { Wide-area augmentation system (WAAS) } \\
\text { Tropospheric delay }\end{array}$ \\
Receiver noise & $\begin{array}{l}\text { Modeled as an exponential function of the } \\
\text { elevation angle } \\
\text { Neglect }\end{array}$ \\
\hline
\end{tabular}

Figures 16 and 17 represent the monitoring value error in the constant velocity and acceleration cases, respectively. The bold solid line in both figures is the threshold for cycle slip detection. The dashed line indicates the 1.8 times the relative position error of the simulation. It can be shown that the dashed lines in Figs. 15 and 16 bound the monitoring value error, so the relationship in Eq. (16) is verified.

Then, by investigating the monitoring value error in Figs. 15 and 16, it is noted that the monitoring value error 


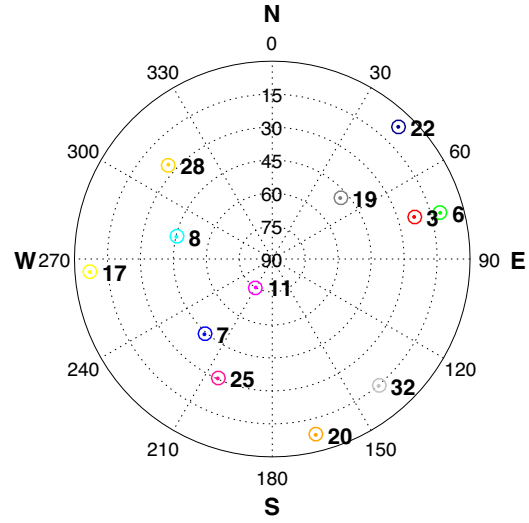

Fig. 15. Sky plot (simulation data)

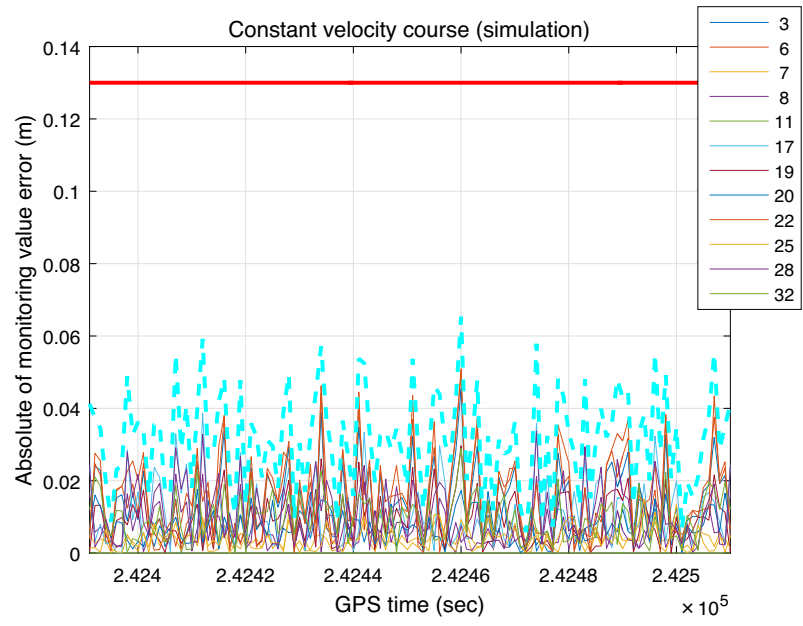

Fig. 16. Monitoring value error (constant velocity case).

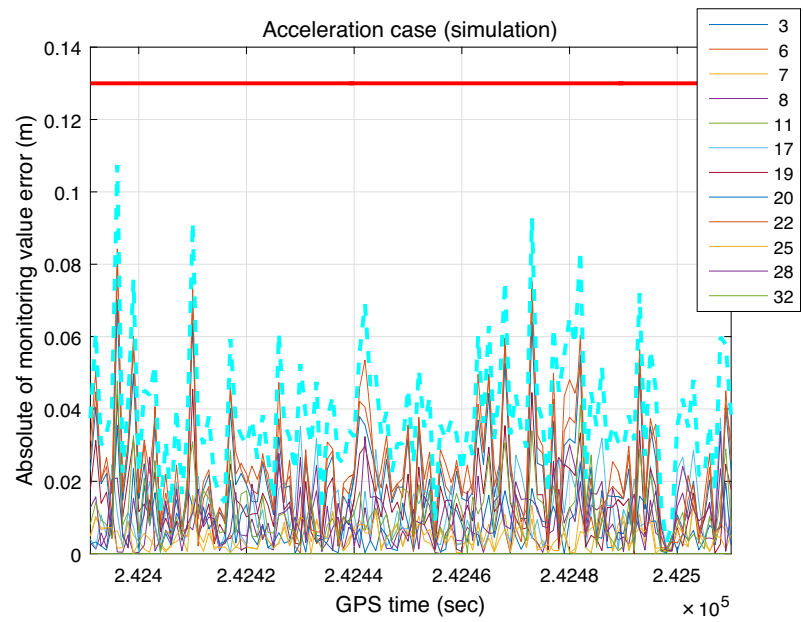

Fig. 17. Monitoring value error (acceleration case).

is also larger in the acceleration case. This is because of the larger relative position estimation error in the acceleration case than in the constant velocity case, which is the same as with the results of the previous section.

In addition, the maximum value of the monitoring value error almost coincides with 1.8 times that of the relative position error. This means that the relative position error con- tributes most significantly to the monitoring value error compared to other error sources.

The resulting monitoring value error in both cases satisfies the requirement for one cycle slip detection established in Section 4. As a result, by bounding the relative position estimation error properly, the monitoring value error performance can be achieved for one cycle slip detection.

\section{Conclusions}

This study suggests a new methodology for choosing an INS as an auxiliary sensor to detect cycle slip. We developed an algorithm from both economic and technical points of view. As a technical issue, we defined the monitoring value for cycle slip detection and analyzed the contribution of error sources. As a result, the INS error contributes most significantly to the monitoring value error. Based on this discovery, we derived the maximum permissible position error caused by the inertial sensor error sources. We considered the bias drift and Gaussian noise of the accelerometer and gyro for the inertial sensor error. By selecting the inertial sensor that produces the maximum permissible position error, we can also achieve cost-effectiveness. Finally, the optimal inertial sensor was selected among the different grades of IMU candidates.

To verify the selection process, we used simulation data. The theoretical derivation results were carefully verified term-by-term. The resulting monitoring value error satisfied the design requirements. Therefore, we concluded that the selected inertial sensor was a suitable device for cycle slip detection, and our suggested algorithm provided an appropriate guideline for choosing an auxiliary sensor.

The results of this study can be used for the design of a low-cost cycle slip detection system for a single-frequency receiver and are also applicable to dual-frequency receivers.

\section{Acknowledgments}

This research was supported by a grant for the "Improvement of GPS Positioning Accuracy of Land Vehicles" funded by LG Electronics, contracted through IVIT at Seoul National University and the program of Development of Space Core Technology through the National Research Foundation of Korea funded by the Ministry of Science, ICT and Future Planning (NRF2015M1A3A3A05027630).

\section{References}

1) Xu, G.: Gps: Theory, Algorithms, and Applications, Springer-Verlag, Berlin Heidelberg, 2007.

2) Realini, E. and Reguzzoni, M.: goGPS: Open Source Software for Enhancing the Accuracy of Low-cost Receivers by Single-frequency Relative Kinematic Positioning, Meas. Sci. Technol., 24, 11 (2013), 115010.

3) Bisnath, S. B. and Langley, R. B.: Automated Cycle-Slip Correction of Dual-Frequency Kinematic GPS Data, Proc. 47th Annu. Conf., Canadian Aeronautics and Space Institute, Ottawa, Canada, 2000, pp. 121-125.

4) Blewitt, G.: An Automatic Editing Algorithm for GPS Data, Geophys. Res. Lett., 17 (1990), pp. 119-202.

5) Gao, Y. and McLellan, J. F.: An Analysis of GPS Positioning Accu- 
racy and Reliability with Dual-Frequency Data, Proc. 9th Int. Tech. Meeting of the Satellite Division of the Institute of Navigation (ION GPS 1996), Kansas City, MO, 1996, pp. 945-951.

6) Gao, Y. and Li, Z.: Cycle Slip Detection and Ambiguity Resolution Algorithms for Dual-Frequency GPS Data Processing, Marine Geodesy, 22 (1999), pp. 169-181.

7) Banville, S. and Langley, R. B.: Mitigating the Impact of Ionospheric Cycle Slips in GNSS Observations, J. Geodesy, 87 (2013), pp. 179-193.

8) Cai, C. S., Liu, Z. Z., Xia, P. F., and Dai, W. J.: Cycle Slip Detection and Repair for Undifferenced GPS Observations under High Ionospheric Activity, GPS Solutions, 17 (2013), pp. 247-260.

9) Liu, Z. Z.: A New Automated Cycle Slip Detection and Repair Method for a Single Dual-Frequency GPS Receiver, J. Geodesy, 85 (2011), pp. 171-183.

10) Altmayer, C.: Enhancing the Integrity of Integrated GPS/INS System by Cycle Slip Detection and Correction, Proc. IEEE Intelligent Vehicles Symp., Dearborn, MA, 2000, pp. 174-179.

11) Colombo, O. L., Bhapkar, U. V., and Evans, A. G.: Inertial-Aided Cycle-Slip Detection/Correction for Precise, Long-Baseline Kinematic GPS, Proc. 12th Int. Tech. Meeting of the Satellite Division of the Institute of Navigation (ION GPS 1999), Nashville, TN, 1999, pp. 1915-1922.

12) Takasu, T. and Yasuda, A.: Cycle Slip Detection and Fixing by MEMS-IMU/GPS Integration for Mobile Environment RTK-GPS, Proc. 21st Int. Tech. Meeting of the Satellite Division of the Institute of Navigation (ION GNSS 2008), Savannah, GA, 2008, pp. 64-71.

13) Du, S.: An Inertial Aided Cycle Slip Detection and Identification Method for Integrated PPP GPS/MEMS IMU System, Proc. 24th Int. Tech. Meeting of the Satellite Division of the Institute of Navigation (ION GNSS 2011), Portland, OR, 2011, pp. 3183-3191.

14) Du, S. and Gao, Y.: Inertial Aided Cycle Slip Detection and Identifica- tion for Integrated PPP GPS and INS, Sensors, 12 (2012), pp. 14344-14362

15) Song, J., Kim, Y., Park, B., Yun, H., and Kee, C.: Predictions of Allowable Sensor Error Limit for Cycle-Slip Detection, T. Jpn. Soc. Aeronaut. Space Sci., 57 (2014), pp. 169-178.

16) Park, B.: A Study on Reducing Temporal and Spatial Decorrelation Effect in GNSS Augmentation System: Consideration of the Correction Message Standardization, Ph.D. Dissertation, Dept. Mech. and Aero. Eng., Seoul National University, Seoul, South Korea, 2007.

17) Song, J., Kim, Y., Park, B., Yun, H., and Kee, C.: Cycle Slip Detection Algorithm by Integrating Vehicle and Inertial Sensors for Land Vehicle Users, Int. Global Navigation Satellite Systems Society IGNSS Symp. 2011, Sydney, Australia, 2011.

18) Flenniken, W.: Modeling Inertial Measurement Units and Analyzing the Effect of Their Errors in Navigation Applications, M.S. Dissertation, University of Auburn, AL, USA, 2005.

19) Xing, Z.: Over-bounding Integrated INS/GNSS Output Errors, Ph.D. Dissertation, Dept. Aero. and Mech. Eng., University of Minnesota, Minnesota, USA, 2010.

20) Park, Y., Kang, C., Lee, D., and Park, C.: A Theoretical Approach to Observability Analysis of the SDINS/GPS in Maneuvering with Horizontal Constant Velocity, Int. J. Control Autom. Syst., 10 (2012), pp. 298-307.

21) Rhee, I., Abdel-Hafez, M. F., and Speyer, J. L.: Observability of an Integrated GPS/INS during Maneuvers. Aerospace and Electronic Systems, IEEE Trans. Aerospace Electronic Syst., 40 (2004), pp. 526-535.

J. Zhang Associate Editor 